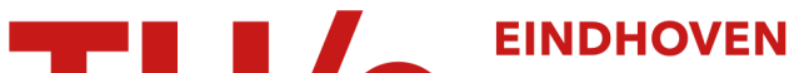 \\ UNIVERSITY OF \\ TECHNOLOGY
}

\section{Stochastic networked control systems with dynamic protocols}

Citation for published version (APA):

Guerreiro Tomé Antunes, D. J., Hespanha, J. P., \& Silvestre, C. J. (2015). Stochastic networked control systems with dynamic protocols. Asian Journal of Control, 17(1), 99-110. https://doi.org/10.1002/asjc.841

DOI:

10.1002/asjc.841

Document status and date:

Published: 01/01/2015

Document Version:

Publisher's PDF, also known as Version of Record (includes final page, issue and volume numbers)

Please check the document version of this publication:

- A submitted manuscript is the version of the article upon submission and before peer-review. There can be important differences between the submitted version and the official published version of record. People interested in the research are advised to contact the author for the final version of the publication, or visit the $\mathrm{DOI}$ to the publisher's website.

- The final author version and the galley proof are versions of the publication after peer review.

- The final published version features the final layout of the paper including the volume, issue and page numbers.

Link to publication

\section{General rights}

Copyright and moral rights for the publications made accessible in the public portal are retained by the authors and/or other copyright owners and it is a condition of accessing publications that users recognise and abide by the legal requirements associated with these rights.

- Users may download and print one copy of any publication from the public portal for the purpose of private study or research.

- You may not further distribute the material or use it for any profit-making activity or commercial gain

- You may freely distribute the URL identifying the publication in the public portal.

If the publication is distributed under the terms of Article $25 \mathrm{fa}$ of the Dutch Copyright Act, indicated by the "Taverne" license above, please follow below link for the End User Agreement:

www.tue.nl/taverne

Take down policy

If you believe that this document breaches copyright please contact us at:

openaccess@tue.nl

providing details and we will investigate your claim. 


\title{
STOCHASTIC NETWORKED CONTROL SYSTEMS WITH DYNAMIC PROTOCOLS
}

\author{
Duarte Antunes, João P. Hespanha, and Carlos Silvestre
}

\begin{abstract}
We consider networked control systems in which sensors, controllers, and actuators communicate through a shared network that introduces stochastic intervals between transmissions, delays, and packet drops. Access to the communication medium is mediated by a protocol that determines which node (one of the sensors, one of the actuators, or the controller) is allowed to transmit a message at each sampling/actuator-update time. We provide conditions for mean exponential stability of the networked closed loop in terms of matrix inequalities, both for investigating the stability of given protocols, such as static round-robin protocols and dynamic maximum error first-try once discard protocols, and conditions to design new dynamic protocols. The main result entailed by these conditions is that, if the networked closed loop is stable for a static protocol, then we can provide a dynamic protocol for which the networked closed loop is also stable. The stability conditions also allow for obtaining an observer-protocol pair that reconstructs the state of a linear time invariant plant in a mean exponential sense and for less conservative stability results than other conditions previously appearing in the literature.
\end{abstract}

Key Words: Networked control systems, dynamic protocols, scheduling, stochastic systems.

\section{INTRODUCTION}

The proliferation of network communication systems in recent years has paved the way for important research in the area of networked control systems. This research area addresses control loops closed via a shared network that provides the medium for sensor, actuator, and controller nodes to communicate.

Walsh and co-authors [1] made strides in the analysis of control systems closed via a local area network, such as a controller-area network, an ethernet, and wireless 802.11 networks. The key assumptions in [1] are that there exists a bound on the interval between transmissions denoted by maximum allowable transfer interval (MATI), and that transmission delays and packet drops are negligible. In [1], an emulation set-up is considered in the sense that the controller

Manuscript received May 30, 2012; revised November 28, 2012; accepted June 27, 2013.

D. Antunes is with the Hybrid and Networked Systems Group, Department of Mechanical Engineering, Eindhoven University of Technology, the Netherlands (corresponding author, e-mail: D.Antunes@tue.nl).

João P. Hespanha is with the Dept. of Electrical and Computer Eng., University of California, Santa Barbara, CA 93106-9560, USA. (e-mail: hespanha@ece.ucsb.edu).

C. Silvestre is with the Dep. of Electrical and Comp. Eng. of the Faculty of Science and Technology of the University of Macau, Taipa, Macau on leave from the Dep. of Electrical Eng. and Computer Science, Instituto Superior Técnico, ISR, 1046-001 Lisboa, Portugal. (e-mail: cjs@isr.ist.utl.pt).

This work is supported by the Dutch Science Foundation (STW) and the Dutch Organization for Scientific Research (NWO) under the VICI grant "Wireless controls systems: A new frontier in Automation" (No. 11382), by the European 7th Framework Network of Excellence by the project "Highly-complex and networked control systems (HYCON2-257462), by the NSF CNS-0720842, by project FCT [PEst-OE/ EEI/LA0009/2011], and by the EU Project TRIDENT (Contract No. 248497). for the networked control system is obtained from a previously designed stabilizing continuous-time controller. Two basic types of protocols have been proposed: static protocols, such as the round-robin (RR) protocol, where nodes take turns transmitting data in a periodic fashion, and dynamic protocols, such as the maximum error first-try once discard (MEF-TOD) protocol, where the node that has the top priority in using the communication medium is the one whose current value to transmit differs the most from the last transmitted value. Under this setup, one can attempt to provide an upper bound on the MATI for which stability can be guaranteed. Since these protocols have been proposed in the papers referenced above, MATI bounds have been improved [2-4]. Although [1] illustrated through simulations that using the MEF-TOD protocol allows for preserving stability of the networked closed loop for a larger MATI than that obtained when using the RR protocol, similar conclusions are obtained in [2-4] from sufficient stability conditions, no analytical result has been established proving that this holds in general.

As mentioned in [1], the occurrence of transmission events on the network is often model more appropriately as a random process. This feature is taken into account in [5], which considers networked control systems with MEF-TOD and RR protocols, and with independent and identically distributed (i.i.d.) intervals between transmissions. It is shown that stability can be guaranteed for distributions of the intertransmission intervals that have a support larger than previously derived deterministic upper bounds for the MATI [1-3]. The conservativeness of these results for linear networked control systems using the RR protocol was eliminated in [6]. 
Recently, [7] addressed a model of networked control systems with i.i.d. intervals between transmissions and stochastic delays for a class of quadratic protocols that is more general than MEF-TOD. Through a convex over-approximation approach, sufficient conditions were given for mean exponential stability. In [8], a method was proposed to design an observer-protocol pair to asymptotically reconstruct the states of a linear time invariant (LTI) plant, where the plant outputs are sent through a network with constant intervals between transmissions. The protocol to be designed can be viewed as a weighted version of the MEF-TOD.

In the present paper, we follow this line of research considering that the network imposes i.i.d. intervals between transmissions. We also take into account stochastic delays modeled as in [7] and packet drops. We consider that access to the network is mediated by a dynamic protocol specified as follows. Associated to each node, there is a set of quadratic state functions, which are evaluated at a given transmission time. The node allotted permission to transmit is the one corresponding to the least value of these quadratic state functions. These protocols are more general than the quadratic protocols considered in [7]; thus they are more general than the MEF-TOD protocol.

We establish two stability results for the networked control system. Both of these provide conditions in terms of linear matrix inequalities (LMIs) for investigating the stability in a mean exponential sense of given protocols and provide conditions in terms of BMIs to design quadratic state functions, specifying the dynamic protocol, that yield the networked closed loop stable. The first stability result allows one to prove that, if the networked closed loop is stable for a static protocol, then we can provide a dynamic protocol for which the networked closed loop is also stable. This is the main contribution of this paper and gives an analytical justification on why one should utilize dynamic protocols rather than static, while, e.g., in [1], this conclusion is only illustrated through simulation. The second stability result allows us to extend the work [8] to the case where transmission intervals are stochastic. We also address the relation of this stability result with the necessity of existence of a quadratic stochastic Lyapunov function that assures stability for the networked control system.

We illustrate through benchmark examples that the conditions in this paper are significantly less conservative than other conditions previously appearing in the literature.

A preliminary version of the results presented here appeared in a conference paper [9]. Besides including all of the formal proofs of the results, here we establish the connection between the second of our two main stability results and the existence of a quadratic stochastic Lyapunov function that assures stability for the networked control system.

The remainder of the paper is organized as follows. The networked control problem setup is given in Section II.
The main results are stated in Section III. In Section IV, we compare our results with previous works. Concluding remarks are given in Section V. The proofs of the results are provided in the Appendix.

Notation. We denote by $I_{n}$ and $O_{n}$ the $n \times n$ identity and zero matrices, respectively, and by $\operatorname{diag}\left(\left[A_{1} \ldots A_{n}\right]\right)$ a block diagonal matrix with blocks $A_{i}$. For dimensionally compatible matrices $A$ and $B$, we define $(A, B):=\left[A^{\top} B^{\top}\right]^{\top}$.

\section{PROBLEM FORMULATION}

We start by introducing the networked control stability problem before showing that it can be cast into analyzing the stability of an impulsive system.

\subsection{Networked control setup}

We consider a networked control system for which sensors, actuators, and a controller are connected through a communication network, possibly shared with other users. The plant and controller are described by the following statespace model.

$$
\begin{aligned}
& \text { Plant: } \quad \dot{x}_{P}=A_{P} x_{P}+B_{P} \hat{u}, \quad y=C_{P} x_{P} \\
& \text { Controller: } \dot{x}_{C}=A_{C} x_{C}+B_{C} \hat{y}, \quad u=C_{C} x_{C}+D_{C} \hat{y} .
\end{aligned}
$$

Following an emulation approach, we assume that the controller has been designed to stabilize the closed loop when the process and the controller are directly connected, i.e., $\hat{u}(t)=u(t), \hat{y}(t)=y(t), t \geq 0$, and we are interested in analyzing the effects of the network on the stability of the closed loop. Note that this assumption implies that $\left(A_{P}, C_{P}\right)$ is detectable and that $\left(A_{P}, B_{P}\right)$ is stabilizable. We denote the times at which a node transmits a message by $\left\{t_{k}, k \in \mathbb{N}\right\}$, and we assume that $\hat{u}$ and $\hat{y}$ are held constant between transmission times, i.e.,

$$
\hat{u}(t)=\hat{u}\left(t_{k}\right), \quad \hat{y}(t)=\hat{y}\left(t_{k}\right), \quad t \in\left[t_{k}, t_{k+1}\right), \quad k \in \mathbb{Z}_{\geq 0} .
$$

We denote by $e$ the error signal between the process output and controller input $(\hat{y}-y)$ and between the controller output and process input $(\hat{u}-u)$. In particular,

$$
e:=(\hat{y}-y, \hat{u}-u)
$$

We assume that, while $m:=n_{y}+n_{u}$ nodes compete for the network, where $n_{y}$ and $n_{u}$ denote the number of sensor and actuator nodes, respectively, only one of them is allowed to transmit at each given transmission time. Hence, in our terminology, a single transmitting node can be associated with several entries of the process output $y$ or with several entries of the controller output $u$. We partition the process output vector as $y=\left(y_{1}, \ldots, y_{n_{y}}\right)$, the controller output vector as 


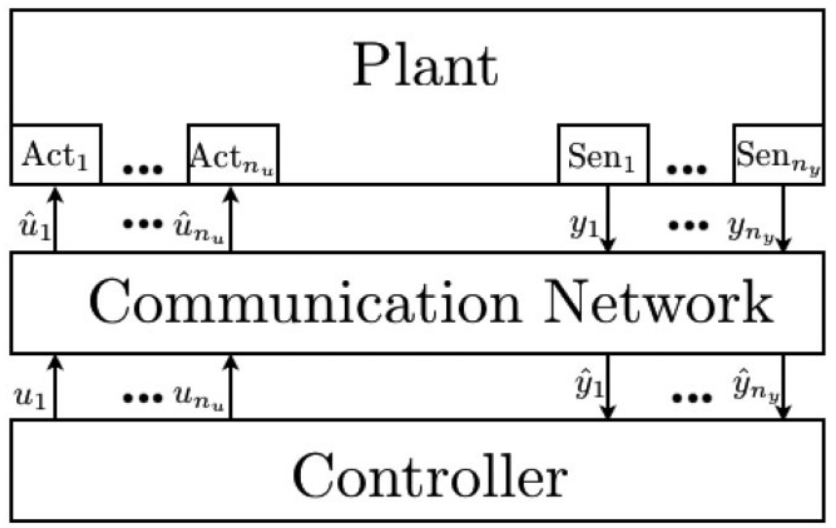

Fig. 1. Networked control setup.

$u=\left(u_{1}, \ldots, u_{n_{u}}\right)$, and the error vector as $e=\left(e_{1}, \ldots, e_{m}\right)$, where each $e_{i}(t) \in \mathbb{R}^{s_{i}}$ is the error associated with node $i \in M:=\{1, \ldots, m\}$, i.e., $e_{i}$ corresponds to a sensor node $y_{i}$ if $i \in\left\{1, \ldots, n_{y}\right\}$ and to an actuator node $u_{i-n_{y}}$ if $i \in\left\{n_{y}+1\right.$, $\left.\ldots, n_{y}+n_{u}\right\}$. The setup is shown in Figure 1. The state of the networked control system is defined by the vector $x:=\left(x_{P}, x_{C}\right.$, $e$ ), where $x_{P} \in \mathbb{R}^{n_{P}}, x_{C} \in \mathbb{R}^{n_{C}}, e \in \mathbb{R}^{n_{e}}$, and $x \in \mathbb{R}^{n_{x}}$. We are interested in scenarios for which the following assumptions hold:

(i) The time intervals $\left\{h_{k}:=t_{k+1}-t_{k}\right\}$ are i.i.d. described by a probability measure $\mu$ with support on $[0, \gamma]$, $\gamma \in \mathbb{R}_{\geq 0} \cup\{+\infty\}$, i.e., $\operatorname{Prob}\left(a \leq h_{k} \leq b\right)=\int_{a}^{b} \mu(d r)$ for $a, b \in[0, \gamma]$.

(ii) Corresponding to a transmission at time $t_{k}$ there is a transmission delay $d_{k}$ no greater than $h_{k}=t_{k+1}-t_{k}$; A joint stationary probability density $\chi$ describes $\left(h_{k}, d_{k}\right)$, in the sense that

$$
\operatorname{Prob}\left(a \leq h_{k} \leq b, c \leq d_{k} \leq d\right)=\int_{a}^{b} \int_{c}^{d} \chi(d r, d s)
$$

where $a, \quad b \in[0, \quad \gamma]$ and $\operatorname{Prob}\left(a \leq h_{k} \leq b\right.$, $\left.c \leq d_{k} \leq d\right)=0$ if $c>b$. In view of (i) and (ii), we see that $\mu([a, b])=\chi([a, b],[0, b])$.

(iii) At each transmission time, there is a probability $p_{\text {drop }}$ that a packet may not arrive at its destination or that it may arrive corrupted (packet drop).

(iv) The nodes implement one of the two protocols:

Dynamic protocol (DP): This protocol is specified by $m_{D}$ symmetric matrices $\left\{R_{i}, i \in \mathcal{M}_{D}\right\}$, $\mathcal{M}_{D}:=\left\{1, \ldots m_{D}\right\}, m_{D} \geq m$. A subset of these matrices $\left\{R_{i}, i \in I_{j}\right\}$ is associated with node $j \in \mathcal{M}$ where $I_{j}:=\left\{i_{1}^{j}, i_{2}^{j}, \ldots, i_{r_{j}}^{j}\right\}$ is an index subset of $\mathcal{M}_{D}$. These subsets are assumed to be nonempty, i.e., $r_{j} \geq 1$, disjoint, and the $r_{j}$ are such that $\sum_{j=1}^{m} r_{j}=m_{D}$. The node $j$ allotted to transmit at $t_{k}$ is determined by the map d: $\mathbb{R}^{n_{x}} \mapsto \mathcal{M}$,

$$
\mathrm{d}\left(x\left(t_{k}^{-}\right)\right)=\mathrm{d}_{1} \circ \mathrm{d}_{2}\left(x\left(t_{k}^{-}\right)\right),
$$

where $\mathrm{d}_{2}: \mathbb{R}^{n_{x}} \mapsto \mathcal{M}_{D}$ is given by

$$
\mathrm{d}_{2}\left(x\left(t_{k}^{-}\right)\right):=\operatorname{argmin}_{i \in \mathcal{M}_{D}} x\left(t_{k}^{-}\right)^{\top} R_{i} x\left(t_{k}^{-}\right),
$$

and $\mathrm{d}_{1}: \mathcal{M}_{D} \mapsto \mathcal{M}$ is given by

$$
\mathrm{d}_{1}(i):=\left\{j: i \in I_{j}\right\}
$$

In case the minimum in (7) is achieved simultaneously for several values of the index $i$, stability of the networked control system should be guaranteed regardless of the specific choice for the argmin. In view of (6), the error $e$ is updated at time $t_{k}$ according to

$$
e\left(t_{k}\right)=\left(I_{n_{e}}-\Lambda_{\mathrm{d}\left(x\left(t_{\bar{k}}\right)\right)}\right) e\left(t_{k}^{-}\right),
$$

where $\quad \Lambda_{j}:=\operatorname{diag}\left(\left[0_{\sum_{i=1}^{j-1} s_{i}} I_{s_{j}} 0_{\sum_{i=j+1}^{m} s_{i}}\right]\right), j \in \mathcal{M}$. That is, only the components of $\hat{y}$ or $\hat{u}$ associated with the node that transmits are updated by the corresponding components of $y\left(t_{k}^{-}\right)$or $u\left(t_{k}^{-}\right)$. We call a dynamic protocol regular if for every $j \in \mathcal{M}_{D}$ there exists a state $x$ such that $j$ is the unique index such that $j=\operatorname{argmin}_{i \in \mathcal{M}_{D}} x^{\top} R_{i} x$. An irregular dynamic protocol can always be made regular by discarding unnecessary matrices $R_{i}$.

Static Protocol (SP): The nodes transmit in a $m_{s}$-periodic sequence determined by a periodic function

$$
\mathrm{s}: \mathbb{N} \mapsto \mathcal{M}
$$

with period $m_{S}$. In this case, the error $e$ is updated at time $t_{k}$ according to

$$
e\left(t_{k}\right)=\left(I_{n_{e}}-\Lambda_{\mathrm{s}(k)}\right) e\left(t_{k}^{-}\right) .
$$

We assume that $\mathrm{s}$ is onto, i.e., each node transmits at least once in a period. When $m_{S}=m$, each node transmits exactly once in a period.

As mentioned in Section I, Assumptions (i)-(iii) are appropriate for networked control systems in which feedback loops are closed via local area networks $(c f .[1,10])$. In particular, Assumption (i) holds for scenarios in which nodes attempt to do periodic transmissions of data, but these regular transmissions may be perturbed by the medium access. It is typically the case in carrier sense multiple access (CSMA) protocols that nodes may be forced to back off for a typically random amount of time until the network becomes available. Note that networks with CSMA protocols protocol, such as the ethernet of wireless 802.11, are prevalent in modern communication systems. The probability distribution of the time interval between transmissions, which can be estimated experimentally or by running Monte Carlo simulations of the 
protocol, is determined by two factors: the congestion of the network and the delay introduced by the medium access protocol.

The class of dynamic protocols that we describe in (iv) allows a node to transmit if the state of the networked control system lies in a given region of the state space, partitioned according to quadratic restrictions. This class of protocols boils down to the quadratic protocols introduced in [7] when $m_{D}=m$. Thus, our definition allows for ampler partitions of the state-space than quadratic protocols, and, as we shall see, it also allows us to see that dynamic protocols are in a sense better than static ones. If we make $m_{D}=m$ and choose $P>0$ such that $R_{i}=P-\operatorname{diag}\left(\left[0_{n_{P}+n_{C}} \Lambda_{i}\right]\right)>0$, then (6) becomes the usual MEF-TOD protocol, where the node that transmits is the one with the maximum norm of the error $e_{i}(t)$ between its current value and its last transmitted value.

\subsection{Impulsive systems}

Suppose that there are no delays, i.e. $d_{k}=0$, and no packet drops, i.e., $p_{\text {drop }}=0$. Then, we can write the networked control system (1), (2), (3), (4), in the form of the following impulsive system:

$$
\begin{gathered}
\dot{x}(t)=A x(t), \quad t \in \mathbb{R}_{\geq 0}, t \neq t_{k} \\
x\left(t_{k}\right)=J_{\mathrm{p}\left(x\left(t_{k}^{-}\right), k\right)} x\left(t_{k}^{-}\right), \quad k \in \mathbb{N}_{0}
\end{gathered}
$$

where $x \in \mathbb{R}^{n_{x}}, x\left(0^{-}\right):=x_{0}$, and $t_{k+1}-t_{k}$ are i.i.d. random variables characterized by the probability density $\mu$, and where the map $\mathrm{p}$ takes the following form for dynamic and static protocols

$$
\begin{array}{ll}
\text { DP: } & \mathrm{p}\left(x\left(t_{k}^{-}\right), k\right)=\mathrm{d}\left(x\left(t_{k}^{-}\right)\right) \\
\text {SP: } & \mathrm{p}\left(x\left(t_{k}^{-}\right), k\right)=\mathrm{s}(k) .
\end{array}
$$

For example, the following expressions for $A$ and $\left\{J_{i}, i \in \mathcal{M}\right\}$ correspond to the case in which the controller and plant are directly connected and only the outputs are transmitted through the network, i.e., $\hat{u}(t)=u(t), x=\left(x_{p}, x_{C}, \hat{y}-y\right)$.

$$
\begin{aligned}
A & =\left[\begin{array}{ll}
A_{11} & A_{12} \\
A_{21} & A_{22}
\end{array}\right] \\
A_{11} & =\left[\begin{array}{cc}
A_{P}+B_{P} D_{C} C_{P} & B_{P} C_{C} \\
B_{C} C_{P} & A_{C}
\end{array}\right] \\
A_{12} & =\left[\begin{array}{c}
B_{P} D_{C} \\
B_{C}
\end{array}\right] \\
A_{21} & =-\left[\begin{array}{ll}
C_{P} & 0
\end{array}\right] A_{11} \\
A_{22} & =-\left[\begin{array}{ll}
C_{P} & 0
\end{array}\right] A_{12} \\
J_{i} & =\operatorname{diag}\left(\left[I_{n_{P}+n_{C}} I_{n_{e}}-\Lambda_{i}\right]\right), \quad i \in \mathcal{M} .
\end{aligned}
$$

This case will be considered in Section IV. Expressions for the general case considered in Section II can be obtained (see, e.g., $[11$, p. 5]).

To take into account delays and packet drops modeled as described in Section II, we consider the following impulsive system:

$$
\begin{aligned}
\dot{x}(t) & =A x(t), \quad t \in \mathbb{R}_{\geq 0}, \quad t \neq t_{k} \\
x\left(t_{k}\right) & =K_{\mathrm{p}\left(x\left(t_{k}\right), k\right)}^{q_{k}} x\left(t_{k}^{-}\right), \quad k \in \mathbb{N}_{0} \\
x\left(s_{k}\right) & =\operatorname{Lx}\left(s_{k}^{-}\right), \quad t_{k} \leq s_{k} \leq t_{k+1},
\end{aligned}
$$

where $\mathrm{p}\left(x_{k}, k\right)$ is defined as in (13) for dynamic protocols and as in (14) for static protocols and where the initial condition is set to $x\left(0^{-}\right):=x_{0}$. The random variables $t_{k}$ and $s_{k}$ are defined completely by the inter-sampling times $h_{k}:=t_{k+1}-t_{k}$ and by the delays $d_{k}:=s_{k}-t_{k}$. The $\left(h_{k}, d_{k}\right)$ are i.i.d., and are as described by (5). The $q_{k} \in\left\{1, \ldots, n_{q}\right\}$ are i.i.d., and such that $\operatorname{Prob}\left[q_{k}=j\right]=w_{j} \forall j \in\left\{1, \ldots, n_{q}\right\}, k \geq 0$. Below we provide expressions for $A, L, w_{i}$, and $K_{i}^{j}, i \in \mathcal{M}, j \in\left\{1, \ldots, n_{q}\right\}$, which model the case where the controller and the plant are directly connected and only the plant outputs are transmitted through the network, i.e., $\hat{u}(t)=u(t)$. The state is now considered to be $x=\left(x_{P}, x_{C}, \hat{y}, v\right) \in \mathbb{R}^{n_{x}}$ where $v \in \mathbb{R}^{n_{e}}$ is an auxiliary vector $\left(v_{1}, \ldots v_{m}\right)$ that is updated with the sampled value $v_{j}=y_{j}\left(t_{k}\right)$ at each sampling time $t_{k}$ at which node $j$ is allowed to transmit. Nevertheless, the update only takes place if a packet sent at $t_{k}$ is not dropped and the sampled value $v_{j}$ is used only to update the value of $\hat{y}_{j}$ after a transmission delay $d_{k}$ at time $s_{k}=t_{k}+d_{k}$.

$$
\begin{aligned}
& A=\left[\begin{array}{ccc}
A_{11} & A_{12} & 0 \\
0 & 0 & 0 \\
0 & 0 & 0
\end{array}\right] \\
& A_{11}=\left[\begin{array}{cc}
A_{P} & B_{P} C_{C} \\
0 & A_{C}
\end{array}\right] \\
& A_{12}=\left[\begin{array}{c}
B_{P} D_{C} \\
B_{C}
\end{array}\right] \\
& n_{q}=2, w_{1}=1-p_{\text {drop }}, w_{2}=p_{\text {drop }} \\
& K_{i}^{1}=\left[\begin{array}{cccc}
I_{n P} & 0 & 0 & 0 \\
0 & I_{n_{C}} & 0 & 0 \\
0 & 0 & I_{n_{e}} & 0 \\
\Lambda_{i} C_{P} & 0 & 0 & I_{n_{e}}-\Lambda_{i}
\end{array}\right], \\
& K_{i}^{2}=I_{n_{P}+n_{C}+2 n_{e}}, i \in \mathcal{M} \\
& L=\left[\begin{array}{ccc}
I_{n_{P}+n_{C}} & 0 & 0 \\
0 & 0 & I_{n_{e}} \\
0 & 0 & I_{n_{e}}
\end{array}\right] .
\end{aligned}
$$

Again, the expressions for the general case considered in Section II can be obtained. It is also important to mention that there are other ways to model the setup with delays and 
packet drops described in Section II. For example, one can find a similar model to (16) but introduce the dependency on the variable $q_{k}$ modeling the packet drops in the matrix $L$.

\subsection{Stability notion}

We define stability for System (12) in terms of the following auxiliary system obtained by considering the state of (12) only at times $t_{k}^{\text {: }}$.

$$
z_{k+1}=e^{A h_{k}} J_{\mathrm{p}\left(z_{k}, k\right)} z_{k}, \quad k \in \mathbb{N}_{0},
$$

where $z_{k}:=x\left(t_{k}^{-}\right)$, and $z_{0}=x_{0}$. We say that (12) is mean exponentially stable (MES) if there exist constants $c>0$ and $0<\alpha<1$ such that, for any initial condition $x_{0}$, we have:

$$
\mathbb{E}\left[z_{k}^{\top} z_{k}\right] \leq c \alpha^{k} z_{0}^{\top} z_{0}, \forall_{k \geq 0} .
$$

The same definition of MES is used for System (16). We assume that the following condition holds:

$$
e^{2 \bar{\lambda}(A) t} r(t)<c e^{-\alpha_{1} t} \text { for some } c>0, \alpha_{1}>0 .
$$

where $\bar{\lambda}(A)$ is the real part of the eigenvalues of $A$ with largest real part and $r(t):=\mu((t, \gamma])$ denotes the survivor function. Assuming (20), we were able to prove in [6], considering only static protocols, that (19) is equivalent to the more common notion of mean exponential stability in continuous-time where one requires $\mathbb{E}\left[x(t)^{\top} x(t)\right]$ to decrease exponentially. In the present paper, we make no such assertion, although assuming (20) is still useful (e.g., (20) guarantees that (22) is bounded).

\section{MAIN RESULTS}

For simplicity, we assume in Subsections 3.1 and 3.2, that there are no delays, i.e., $d_{k}=0, \forall_{k}$, and no packet drops, i.e., $p_{\text {drop }}=0$, and we consider the general case in Subsection 3.3.

\subsection{Stability result I and dynamic vs. static protocols}

The following is our first stability result for (12), providing a test for sufficent conditions for (12) to be mean exponentially stable.

Theorem 1. The system (12) with dynamic protocol (13) is MES if there exist scalars $\left\{0 \leq p_{j i} \leq 1, j, i \in \mathcal{M}_{D}\right\}$ with $\sum_{j=1}^{m_{D}} p_{j i}=1, \forall_{i \in \mathcal{M}_{D}}$ and $n_{x} \times n_{x}$ symmetric matrices $\left\{R_{i}>0, i \in \mathcal{M}_{D}\right\}$ such that:

$$
J_{\mathrm{d}_{1}(i)}^{\top}\left(\sum_{j=1}^{m_{D}} p_{j i} E\left(R_{j}\right)\right) J_{\mathrm{d}_{1}(i)}-R_{i}<0, \forall_{i \in \mathcal{M}_{D}},
$$

where

$$
E\left(R_{j}\right):=\int_{0}^{\gamma} e^{A^{\top} h} R_{j} e^{A h} \mu(d h)
$$

This result can be used to analyze if a given protocol yields the networked control system stable or to synthesize a protocol that achieves this.

Analysis. Note first that a given dynamic protocol specified by $R_{i}>0, i \in \mathcal{M}_{D}$ is equivalent to a dynamic protocol specified by

$$
\tilde{R}_{i}=P+R_{i}>0, i \in \mathcal{M}_{D},
$$

where $P$ can be any symmetric matrix such that $P+R_{i}>0$. If we replace in (21) the matrices $R_{i}$ by $\tilde{R}_{i}$, given by (23), we obtain that (21), (23) are LMIs in the variables $P$ and $p_{j i}$ (using the fact that $\left.\sum_{j=1}^{m} p_{j i}=1, \forall_{i \in \mathcal{M}_{D}}\right)$.

Synthesis. If we allow $R_{i}$ to be variables in (21), then (21) are basically BMIs. In fact, if we chose a basis $B_{l}$ for the linear space of symmetric matrices, we have $R_{i}=\sum_{l=1}^{n_{s}} b_{i l} B_{l}$ and (21) depends on the products $p_{j i} b_{i l}$. In this case, the dynamic protocol, determined by the matrices $R_{i}$, comes out from the solution to (21).

Remark 2. Stability condition (21) resembles a stability condition for Markov jump linear systems [12]. Indeed, suppose that we choose protocol $\mathrm{p}$ for (12) according to the following stochastic rule:

$$
\begin{aligned}
& \operatorname{Prob}\left[\omega_{k+1}=j \mid \omega_{k}=i\right]=\mu_{i j}, i, j \in \mathcal{M}_{D} \\
& \mathrm{p}\left(x\left(t_{k}^{-}\right), k\right)=\mathrm{d}_{1}\left(\omega_{k}\right), \quad k \geq 0,
\end{aligned}
$$

where $\omega_{k}$ is a Markov chain with $m_{D} \geq m$ states, $\sum_{j=1}^{m D} \mu_{j i}=1, \forall_{i \in \mathcal{M}_{D}}$, and $\mathrm{d}_{1}$ is described by (8). Then, it is known (cf. [12, ch. 4]) that (18) with scheduling (24) is mean square stable $\left(\lim _{k \rightarrow \infty} \mathbb{E}\left[x\left(t_{k}^{-}\right)^{\top} x\left(t_{k}^{-}\right)\right]=0, \forall_{x_{0}}\right)$ if and only if there exist $n_{x} \times n_{x}$ symmetric matrices $\left\{R_{i}>0, i \in \mathcal{M}_{D}\right\}$ such that (21) holds for $p_{j i}=\mu_{i j}, \forall i, j \in \mathcal{M}_{D}$ Note, however, that contrary to Protocol (24), Protocol (6), (8) is a deterministic state-dependent protocol and Theorem 1 assures stability in the deterministic sense (19).

To state the next theorem, we need the following result, which provides necessary and sufficient stability conditions under which (12) is mean exponentially stable for a static protocol (14). The proof can be found in [6] and is omitted 
here. Let $\lfloor i+1\rfloor:=i+1$ if $i \in\left\{1, \ldots, m_{S}-1\right\}$ and $\lfloor i+1\rfloor=1$ if $i=m_{S}$. Let $\mathcal{M}_{S}:=\left\{1, \ldots, m_{S}\right\}$.

Theorem 3. The system (12), with static protocol (14) is MES if and only if there exist $n_{x} \times n_{x}$ symmetric matrices $\left\{R_{i}>0, i \in \mathcal{M}_{S}\right\}$ such that:

$$
J_{\mathrm{s}(i)}^{\top} E\left(R_{[i+1]}\right) J_{\mathrm{s}(i)}-R_{i}<0, \forall_{i \in \mathcal{M}_{\mathrm{s}}}
$$

where $E\left(R_{[i+1]}\right)$ is given as in (22).

The following result, which builds upon Theorems 1 and 3, establishes that, if the networked closed loop is stable for a static protocol, then we can provide a dynamic protocol for which the networked closed loop is also stable. This is one of the main contributions of the paper.

Theorem 4. If the networked control system is MES for a static protocol with period $\mathrm{m}_{\mathrm{S}}$ then there exists a dynamic protocol taking the form (6), with $m_{D}=m_{S}$, that yields the networked control system MES.

Proof. Since the stability conditions of Theorem 3 are necessary and sufficient, there exists a static protocol with period $m_{S}$ that yields the networked control system MES if and only if there exist $\left\{R_{i}, i \in \mathcal{M}_{S}\right\}$ such that (25) holds for (12) with matrices defined by (15). This implies that, if we consider a dynamic protocol with $m_{D}=m_{S}$, $I_{j}=\left\{k \in \mathcal{M}_{S}: \mathrm{s}(k)=j\right\}, j \in \mathcal{M}$, then $\mathrm{d}_{1}(i)=\mathrm{s}(i)$, for $i \in \mathcal{M}_{S}$ and (21) holds with

$$
p_{j i}= \begin{cases}1, & \text { if } i<m_{D} \text { and } j=i+1, \\ 1, & \text { if } i=m_{D} \text { and } j=1, \\ 0 & \text { otherwise }\end{cases}
$$

and with $\left\{R_{i}, i \in \mathcal{M}_{S}=\mathcal{M}_{D}\right\}$ taken to be the solution to (25).

From the proof of Theorem 4, we see that the matrices $\left\{R_{i}, i \in \mathcal{M}_{D}\right\}$ that characterize the dynamic protocol mentioned in its statement can be taken to be the solution to (25). Note that, in the special case where $m_{D}=m=m_{S}$, Theorem 4 states that, if there exists a round-robin protocol with period $m_{S}=m$, i.e., each node only transmits exactly once in a period, which yields the networked control system MES, then one can find a quadratic protocols, as introduced in [7], that also yields the networked control system MES.

Remark 5. The fact that the stability conditions of Theorem 3 are necessary and sufficient the key to obtaining Theorem 4 . In [4], a similar reasoning to Theorem 4 can be used to prove that, if the stability conditions provided there for quadratic protocols ( $c f$. [4, Theorem 3]) hold, then so do the stability conditions for a static protocol in the special case where each node transmits only once in a period (cf. [7, Theorem 3]). Nevertheless, since the conditions provided in [4] are only sufficient for the RR protocol, it does not allow one to conclude that, if a stabilizing static protocol exists, then so does a dynamic protocol, as stated in Theorem 4. Although [7] does not explicitly present stability conditions for a static protocol, the same remarks should apply, since convex overapproximations introduce conservativeness.

\subsection{Stability result II and observer-protocol design}

The following is our second stability result for (12). Similar to Theorem 1, this theorem provides testable sufficent conditions for (12) to be mean exponentially stable. Nevertheless, it will allow us to obtain different results than Theorem 1.

Theorem 6. The system (12) with dynamic protocol (13) is MES if there exist an $n_{x} \times n_{x}$ symmetric matrix $W>0$, scalars $\quad\left\{c_{i j} \geq 0, i, j \in \mathcal{M}_{D}, i \neq j\right\}$, and $n_{x} \times n_{x}$ matrices $R_{i}, i \in \mathcal{M}_{D}$ such that:

$$
J_{\mathrm{d}(i)}^{\top} E(W) J_{\mathrm{d}(i)}+\sum_{j=1, j \neq i}^{m D} c_{i j}\left(R_{j}-R_{i}\right)-W<0, \forall_{i \in \mathcal{M}_{D}}
$$

where $E(W):=\int_{0}^{\gamma}\left(e^{A h}\right)^{\top} W e^{A h} \mu(d h)$.

Given a quadratic protocol, i.e., specific values for the matrices $R_{i}$, testing if (26) holds is an LMI feasibility problem. To design a protocol for which mean exponential stability of the networked control system is guaranteed, we can take the $\left\{R_{i}, i \in \mathcal{M}_{D}\right\}$ as additional unknowns and (26) should now be viewed as a BMI feasibility problem.

The proof of Theorem 6 builds upon establishing that, if there exists a positive definite matrix $W$, positive constants $c_{i j}$ and matrices $R_{i}$ such that (26) holds, then the quadratic function

$$
V(x):=x^{\top} W x
$$

is a stochastic Lyapunov function for System (18) (which models (12) at sampling times) in the sense that the following condition holds for (18)

$$
\mathbb{E}\left[V\left(z_{k+1}\right)\right]-V\left(z_{k}\right) \leq-z_{k}^{\top} Z z_{k}, \forall_{z_{k} \in \mathbb{R}^{n_{x}}}
$$

for some $Z>0$. The next result shows that, under certain conditions, which include the case $m_{D}=m=2$, i.e., only two nodes pertaining to the closed loop access the network, the converse holds. Let $\operatorname{co}(\mathcal{A})$ denote the convex hull of a set $\mathcal{A}$, and, for each $i \in \mathcal{M}_{D}$, define the function 


$$
g_{0}^{i}(y):=y^{\top}\left(W-J_{\mathrm{d}(i)}^{\top} E(W) J_{\mathrm{d}(i)}\right) y
$$

and the $m_{D}-1$ functions

$$
g_{j}^{i}(y)=y^{\top}\left(R_{j}-R_{i}\right) y, \quad j \in \mathcal{M}_{D}-\{i\} .
$$

Define also the following sets in $\mathbb{R}^{m_{x}}$

$$
\begin{aligned}
\mathcal{K}^{i}:= & \left\{\left(g_{0}^{i}(y), g_{1}^{i}(y), \ldots, g_{i-1}^{i}(y), g_{i+1}^{i}(y), \ldots,\right.\right. \\
& \left.\left.g_{m_{D}}(y)\right) \mid y \in \mathbb{R}^{m_{x}}\right\}, \quad i \in \mathcal{M}_{D},
\end{aligned}
$$

and

$$
\mathcal{N}:=\left\{\left(\eta_{0}, \eta_{1}, \ldots, \eta_{m_{D}}\right) \mid \eta_{0}<0, \quad \eta_{k}>0,1 \leq k \leq m_{D}\right\} .
$$

Theorem 7. Suppose that the dynamic protocol (13) is regular. Then, if

$$
\mathcal{K}^{i} \cap \mathcal{N}=\emptyset \Rightarrow \operatorname{co}\left(\mathcal{K}^{i}\right) \cap \mathcal{N}=\emptyset, \forall_{i \in \mathcal{M}_{D}},
$$

there exists an $n_{x} \times n_{x}$ symmetric matrix $W>0$, scalars $\left\{c_{i j} \geq 0, i, j \in \mathcal{M}_{D}, i \neq j\right\}$, and $n_{x} \times n_{x}$ matrices $R_{i}, i \in \mathcal{M}_{D}$ such that (26) holds if and only if there exists a quadratic stochastic Lyapunov function taking the form (27) such that (28) holds. In particular, (29) holds if $m_{D}=2$.

The proof of Theorem (7) relies on the S-Procedure [13] which is a relaxation technique that can be used to provide stability conditions for linear systems with quadratic constraints. In particular, Condition (29) is a condition for the S-Procedure to be lossless, and it is always satisfied in the case in which there is only one quadratic constraint $\left(m_{D}=2\right)$.

We show next that Theorem 6 allows one to extend the observer-protocol design proposed in [8].

\subsubsection{Observer design}

Suppose that we wish to estimate the state of the following plant

$$
\dot{x}_{P}(t)=A_{P} x_{P}(t), \quad y(t)=C_{P} x_{P}(t), \quad x_{P}(0)=x_{P 0}
$$

where the $m$ outputs $y(t)=\left(y_{1}, \ldots, y_{m}\right), y_{i} \in \mathbb{R}^{s_{i}}$ are sent through a network that imposes i.i.d. intervals between transmissions to a remote observer. As in Section II, we denote by $\mu$ the measure that defines the inter-transmission times $h_{k}=t_{k+1}-t_{k}$ and we let $\mathcal{M}=\{1 \ldots, m\}$. Also let $\Psi_{j}:=\operatorname{diag}\left(\left[0_{s_{1}}, \ldots, I_{s_{j}}, \ldots, 0_{s_{m}}\right]\right)$, for $j \in \mathcal{M}$ A natural model based linear remote observer for this system is defined by

$$
\dot{\hat{x}}(t)=A_{P} \hat{x}(t)+L_{k} \Psi_{\mathrm{c}\left(x_{e}\left(t_{\bar{k}}\right)\right)}\left(C_{P} \hat{x}\left(t_{k}^{-}\right)-y\left(t_{k}^{-}\right)\right),
$$

where the observer gains $L_{k}$ to be designed are allowed to depend on the index $k$ and the map

$$
\mathrm{c}\left(x_{e}\left(t_{k}^{-}\right)\right):=\operatorname{argmin}_{j \in \mathcal{M}} x_{e}\left(t_{k}^{-}\right)^{\top} C_{P}^{\top} S_{j} C_{P} x_{e}\left(t_{k}^{-}\right)
$$

determines which node transmits at $t_{k}$, based on the estimation error $x_{e}\left(t_{k}^{-}\right):=\hat{x}\left(t_{k}^{-}\right)-x_{P}\left(t_{k}^{-}\right)$, where $\left\{S_{j}, j \in \mathcal{M}\right\}$ is a set of $m$ matrices. As argued in [8], the sensors should run a replica of the remote observer to access $\hat{x}(t)$, which allows each node to encode in the message arbitration field $x_{e}\left(t_{k}^{-}\right)^{\top} C_{P}^{\top} S_{j} C_{P} x_{e}\left(t_{k}^{-}\right)$, where $C_{P} x_{e}\left(t_{k}^{-}\right)=C_{P} \hat{x}\left(t_{k}^{-}\right)-y_{j}\left(t_{k}^{-}\right), j \in \mathcal{M}$.

The resulting estimation error $x_{e}:=\hat{x}-x_{P}$ evolves according to

$$
\dot{x}_{e}(t)=A_{P} x_{e}(t)+L_{k} \Psi_{\mathrm{c}\left(x_{e}\left(t_{k}\right)\right)} C_{P} x_{e}\left(t_{k}^{-}\right) .
$$

We can cast this problem into the framework of (12) with dynamic protocol (13) by adding an auxiliary variable $v$ that holds the value of $L_{k} \Psi_{\mathrm{c}\left(x_{e}\left(t_{\bar{k}}\right)\right)} C_{P} x_{e}\left(t_{k}^{-}\right)$in the interval between transmission times $\left.\left[t_{k}, t_{k}+1\right)\right]$, considering $x=\left(x_{e}, v\right)$ and

$$
\begin{aligned}
A & =\left[\begin{array}{ll}
A_{P} & I_{n_{P}} \\
0_{n_{P}} & 0_{n_{P}}
\end{array}\right], \\
J_{i} & =\left[\begin{array}{ll}
I_{n_{P}} & 0_{n_{P}} \\
0_{n_{P}} & 0_{n_{P}}
\end{array}\right]+\left[\begin{array}{c}
0 \\
L_{k}
\end{array}\right]\left[\begin{array}{ll}
\Psi_{i} C_{P} & 0
\end{array}\right], \\
R_{i} & =\left[\begin{array}{cc}
C_{P}^{\top} S_{i} C_{P} & 0 \\
0 & 0_{n_{P}}
\end{array}\right], 1 \leq i \leq m=m_{D} .
\end{aligned}
$$

In the following theorem, we propose a method to obtain observer gains $L_{k}$ that yield the networked control system MES. To state the result we need the following assumption:

$$
H(s):=\int_{0}^{s} e^{A P r} d r \text { is invertible for every } s \in[0, \gamma] .
$$

While this assumption holds for a large class of matrices $A_{P}$, it is possible to construct examples where it does not, as in the case where $\gamma>s=2 \pi$ and $A_{P}=\left[\begin{array}{cc}0 & 1 \\ -1 & 0\end{array}\right]$, in which case $H(s)=0$. Let $n_{y}:=\sum_{i=1}^{m} s_{i}$.

Theorem 8. Suppose that (34) holds. If there exist an $n_{P} \times n_{P}$ symmetric matrix $P>0$, an $n_{y} \times n_{y}$ matrix $Y$, an $n_{P} \times n_{y}$ matrix $M, n_{y} \times n_{y}$ matrices $\left\{S_{i}, i \in \mathcal{M}\right\}$, and scalars $\left\{c_{i j} \geq 0, i, j \in \mathcal{M}\right\}$ such that

$$
\begin{aligned}
& F(P)+D M \Psi_{i} C_{P}+\left(D M \Psi_{i} C_{P}\right)^{\top}+C_{P}^{\top} Y C_{P} \\
& +\sum_{j=1, j \neq i}^{m} c_{i j}\left(C_{P}^{\top} S_{j} C_{P}-C_{P}^{\top} S_{i} C_{P}\right)-P<0, \forall_{i \in \mathcal{M}}
\end{aligned}
$$

$$
\left[\begin{array}{cc}
P & M \\
M^{\top} & Y
\end{array}\right]>0
$$


where $F(P):=\int_{0}^{\gamma} e^{A_{P}^{\top} r} P e^{A P r} \mu(d r)$ and $D:=\int_{0}^{\gamma} e^{A P r} \mu(d r)$, then the observer gain $L_{k}=H\left(h_{k}\right)^{-1} P^{-1} M$ yields (12) with matrices (33) MES.

Note that our proposed observer gain $L_{k}$ depends on the length $h_{k}$ of the time interval $\left\{t_{k+1}-t_{k}\right\}$, which is not known at time $t_{k} \leq t<t_{k+1}(30)$. In practice, this results in a delay in constructing the state estimate that never needs to exceed $h_{k}$ since the state of the remote observer (30) can only be updated with measurement $y\left(t_{k}\right)$ at time $t_{k+1}$, at which $h_{k}$ can be computed.

Similar to Theorem 6 , the conditions of Theorem 8 can be used to investigate the stability of a given protocol determined by matrices $R_{j}$, in which case the problem reduces to an LMI feasibility problem, or they can be used to design a protocol, where one needs to solve a BMI feasibility problem.

Remark 9. When the intervals between transmission are constant, one can show that the stability conditions (35) and (36) are equivalent to the ones given in [8], where such an assumption is made. In this case, the matrices $L_{k}$ do not depend on $k$, and can be computed off-line.

\subsection{Extensions to handle delays and packet drops}

Theorems 6 and 1 can be extended to the case where the network introduces packet drops and delays modeled by (16) with Matrices (17). We state these extensions next.

Theorem 10. System (16) with dynamic protocol (13) is MES if there exist scalars $\left\{0 \leq p_{j i} \leq 1, j, i \in \mathcal{M}_{D}\right\}$, with $\sum_{j=1}^{m_{D}} p_{j i}=1, \forall_{i \in \mathcal{M}_{D}}$, and $n_{x} \times n_{x}$ symmetric matrices $\left\{R_{i}>0, i \in \mathcal{M}_{D}\right\}$ such that:

$$
\sum_{l=1}^{n_{q}} w_{l}\left(K_{\mathrm{d}_{1}(i)}^{l}\left(\sum_{j=1}^{m_{D}} p_{j i} E\left(R_{j}\right)\right) K_{\mathrm{d}_{1}(i)}^{l}\right)-R_{i}<0, \forall_{i \in \mathcal{M}_{D}},
$$

where

$$
E\left(R_{j}\right):=\int_{0}^{\gamma} \int_{0}^{h}\left(e^{A h-s} L e^{A s}\right)^{\top} R_{j} e^{A h-s} L e^{A s} \chi(d h, d s) .
$$

Theorem 11. System (16) with dynamic protocol (13) is MES if there exist an $n_{x} \times n_{x}$ symmetric matrix $W>0$, scalars $\left\{c_{i j} \geq 0, i, j \in \mathcal{M}_{D}, i \neq j\right\}, \quad$ and $n_{x} \times n_{x}$ matrices $R_{i}, i \in \mathcal{M}_{D}$ such that:

$$
\begin{aligned}
& \sum_{l=1}^{n_{q}} w_{l}\left(K_{\mathrm{d}_{1}(i)}^{l}\right)^{\top} E(W) K_{\mathrm{d}_{1}(i)}^{l} \\
& \quad+\sum_{j=1, j \neq i}^{m} c_{i j}\left(R_{j}-R_{i}\right)-W<0, \quad \forall_{i \in \mathcal{M}_{D}},
\end{aligned}
$$

where $E(W)$ is defined as in (37).

\section{NETWORKED CONTROL RESULTS}

In this section, we show that Theorems 6 and 11 reduce the conservatism of the results in [3], [5], and [7]. These three works use the same benchmark problem for the control of a batch reactor, where the plant (1) and controller (2) matrices are given by:

$$
\begin{aligned}
A_{P} & =\left[\begin{array}{cccc}
1.38 & -0.2077 & 6.715 & -5.676 \\
-0.5814 & -4.29 & 0 & 0.675 \\
1.067 & 4.273 & -6.654 & 5.893 \\
0.048 & 4.273 & 1.343 & -2.104
\end{array}\right], \\
B_{P} & =\left[\begin{array}{cc}
0 & 0 \\
5.679 & 0 \\
1.136 & -3.146 \\
1.136 & 0
\end{array}\right], C_{P}=\left[\begin{array}{cccc}
1 & 0 & 1 & -1 \\
0 & 1 & 0 & 0
\end{array}\right] . \\
A_{C} & =\left[\begin{array}{ll}
0 & 0 \\
0 & 0
\end{array}\right], B_{C}=\left[\begin{array}{ll}
0 & 1 \\
1 & 0
\end{array}\right], \\
C_{C} & =\left[\begin{array}{cc}
-2 & 0 \\
0 & 8
\end{array}\right], D_{C}=\left[\begin{array}{cc}
0 & -2 \\
5 & 0
\end{array}\right] .
\end{aligned}
$$

Only the two outputs are sent through the network, i.e., $u(t)=\hat{u}(t)$. The network imposes i.i.d. intervals between transmissions, possibly contains packet drops, and has no delays. The networked control closed loop can be written as in (12), (15) in the absence of drops and as in (16)-(17) when drops occur. Thus, the stability of the networked control system can be tested by Theorems 1, 6, and 10,11. The results are shown in Table I, considering two distributions $\mu$ for the inter-transmissions intervals $h_{k}$ : uniform in the interval $[0, \gamma]$, and exponential with expected value $1 / \lambda_{\text {exp }}$.

From Table I, we can conclude that our results allow a significantly reduction the conservatism of the conditions in [5] and [3] for the same benchmark examples. The results in [7] are very close to the ones obtained with Theorem 1, and both outperform the results obtained with Theorem 6 .

In Table II, we show the results obtained by allowing $R_{i}$ in Theorem 1 to be additional variables, i.e., the protocol is to be designed. Note that Theorem 4 assures that the values obtained with Theorem 1 for the maximum support of a uniform distribution that preserves stability when a dynamic protocol (obtained from solving (21)) is utilized are larger than the ones obtained with the necessary and sufficient conditions provided by Theorem 3 for the static protocol, which matches well with the results in Table II.

\section{CONCLUSIONS AND FUTURE WORK}

We provided stability results for networked control systems with stochastic intervals between transmissions, 
Table I. Stability results for the batch reactor example-MEFTOD and Round Robin protocol. NA stands for Not Available.

\begin{tabular}{lccccc}
\hline & \multicolumn{2}{c}{ Dynamic Protocol } & & \multicolumn{2}{c}{ Static Protocol } \\
\cline { 2 - 3 } \cline { 5 - 6 } & no drops & $p=0.5$ & & no drops & $p=0.5$ \\
\hline Max. $\gamma:$ & & & & \\
$h_{k} \sim$ Uni. $(\gamma)$ & & & & \\
Results from [3] & NA & NA & & NA & NA \\
Results from [5] & 0.0372 & 0.0170 & & 0.0517 & 0.0199 \\
Results from [7] & 0.11 & NA & & NA & NA \\
Ths. 6 and 11 & 0.0550 & 0.024 & & NA & NA \\
Ths. 1 & 0.111 & NA & & NA & NA \\
Th. 3 & NA & NA & & 0.112 & 0.0385 \\
Max. $1 / \lambda_{\text {exp }}:$ & & & & \\
$h_{k} \sim$ Exp $\left(\lambda_{\text {exp }}\right)$ & & & & \\
Results from [3] & 0.0095 & 0.0046 & & NA & NA \\
Results from [5] & 0.0158 & 0.00795 & & 0.0217 & 0.00924 \\
Results from [7] & NA & NA & & NA & NA \\
Ths. 6 and 11 & 0.0226 & 0.01124 & & NA & NA \\
Ths. 1 & 0.0357 & NA & NA & NA \\
Th. 3 & NA & NA & & 0.0417 & 0.0188 \\
\hline
\end{tabular}

Table II. Stability results for the batch reactor example-Protocol design, no packet drops.

\begin{tabular}{lcc}
\hline & Dyn. Prot. Design (Th. 1) & Static Prot. (Th. 3) \\
\hline $\begin{array}{l}\text { Max. } \gamma: \\
h_{k} \sim \text { Uni. }(\gamma)\end{array}$ & 0.140 & 0.112 \\
\hline
\end{tabular}

delays, and packet drops. Our main result was to show that one can analytically prove that dynamic protocols preserve stability for larger sampling intervals between transmission than static protocols; therefore fewer communication and control computations are required for such protocols in networked control systems.

The assumptions on the communication network in the present work were inspired by the most prevalent communication networks (e.g. ethernet and wireless protocols) in which data is transmitted in atomic units called packets. In such networks, packets carry a large number of bits for data; hence bit-rate limitations are not the main feature of interest. Nevertheless, it would be interesting in the future to consider a networked control system with both packet based communication links and communication links in which bit-rate limitations are the main feature of interest. For work considering bit-rate limitations, see [14].

Although we considered here, for simplicity, a disturbance-free model for the plant, other works cope with stochastic disturbance models for the plant [5]. Taking into account disturbances in the model of the plant can also be a direction for future work.

Motivated by recent research [15-17] on $H_{2}$ optimal problems in the context of networked control systems, an additional possible line for future research is to consider related optimal control problems in the scenarios addressed in the present paper.

\section{APPENDIX A. PROOFS}

Proof of Theorem 1. The discrete-time process $z_{k}$, described by (18), can be shown to be a Markov process due to the i.i.d. assumption on $h_{k}$. In particular

$$
\mathbb{E}_{z_{k}}\left[\mathbb{E}_{z_{k+l}}\left[V\left(z_{k+l+m}\right)\right]\right]=\mathbb{E}_{z_{k}}\left[V\left(z_{k+l+m}\right)\right]
$$

for any bounded measurable function $V$, where $\mathbb{E}_{z_{k}}$ denotes expectation given $z_{k}$, i.e., $\mathbb{E}_{z_{k}}[]:.=\mathbb{E}\left[. \mid z_{k}\right]$. If one can find a function $V$ and positive constants $c_{1}, c_{2}, c_{3}$, such that

$$
c_{1}\|z\|^{2} \leq V(z) \leq c_{2}\|z\|^{2}, \forall z \in \mathbb{R}^{n_{x}}
$$

and

$$
\mathbb{E}_{z_{k}}\left[V\left(z_{k+1}\right)\right]-V\left(z_{k}\right) \leq-c_{3}\left\|z_{k}\right\|^{2}, \forall z_{k} \in \mathbb{R}^{n_{x}}
$$

then we can prove that

$$
\mathbb{E}\left[z_{k}^{\top} z_{k}\right] \leq c \alpha^{k} z_{0}^{\top} z_{0}, \forall_{k \geq 0} \text { for some } 0<\alpha<1, c>0 .
$$

which implies MES for (12) according to Definition (19) since $x\left(t_{k}\right)=J_{i} z_{k}$ for some $i \in \mathcal{M}$. In fact, if (39) and (40) hold, then

$$
E_{z_{k}}\left[V\left(z_{k+1}\right)\right] \leq \alpha V\left(z_{k}\right)
$$

where $0<\alpha=1-\frac{c_{3}}{c_{2}}<1$ must be greater than zero since $V$ is positive. From (38) and (42), we can conclude that

$$
E_{z_{0}}\left[V\left(z_{k}\right)\right] \leq \alpha^{k} V\left(z_{0}\right) .
$$

From (39) and (43), we obtain:

$$
E_{z_{0}}\left[\left\|z_{k}\right\|^{2}\right] \leq \frac{c_{2}}{c_{1}} \alpha^{k}\left\|z_{0}\right\|^{2}, k \geq 0
$$

Take $V\left(z_{k}\right):=\min _{i \in \mathcal{M}_{D}} z_{k}^{\top} R_{i} z_{k}$, which satisfies (39) since $R_{i}>0 \quad \forall_{i \in Q}$. Suppose that $z_{k}$ is such that $i=\mathrm{d}_{2}\left(z_{k}\right)=\operatorname{argmin}_{j \in \mathcal{M}_{D}} z_{k}^{\top} R_{j} z_{k}$, i.e., $V\left(z_{k}\right)=z_{k}^{\top} R_{i} z_{k}$. Note that, in the case where the minimum is achieved simultaneously for several values of the index $i$, any of these indices can be chosen without affecting the present proof. Then, for any $p_{j i} \geq 0: \sum_{j=1}^{m D} p_{j i}=1$, we have: 


$$
\begin{aligned}
\mathbb{E}_{z_{k}}\left[V\left(z_{k+1}\right)\right] & =\mathbb{E}_{z_{k}}\left[\min _{j \in \mathcal{M}_{D}} z_{k}^{\top} J_{\mathrm{d}_{1}(i)}^{\top} e^{A^{\top} h} R_{j} e^{A h} J_{\mathrm{d}_{1}(i)} z_{k}\right] \\
& \leq \mathbb{E}_{z_{k}}\left[\sum_{j=1}^{m_{D}} p_{j i} z_{k}^{\top} J_{\mathrm{d}_{1}(i)}^{\top} e^{A^{\top} h} R_{j} e^{A h} J_{\mathrm{d}_{1}(i)} z_{k}\right] \\
& =z_{k}^{\top} J_{\mathrm{d}_{1}(i)}^{\top} \sum_{j=1}^{m_{D}} p_{j i} E\left(R_{j}\right) J_{\mathrm{d}_{1}(i)} z_{k}
\end{aligned}
$$

Suppose we choose $p_{j i}$ such that (21) holds, i.e.,

$$
J_{\mathrm{d}_{1}(i)}^{\top} \sum_{j=1}^{m_{D}} p_{j i} E\left(R_{j}\right) J_{\mathrm{d}_{1}(i)}-R_{i}=-Q_{i}
$$

for some $Q_{i}>0$. Then, from (44), we conclude that $\mathbb{E}_{z_{k}}\left[V\left(z_{k+1}\right)\right]-V\left(z_{k}\right) \leq-z_{k}^{\top} Q_{i} z_{k}, i=\mathrm{d}_{2}\left(z_{k}\right)$, which implies (40) and concludes the proof.

Proof of Theorem 6. As in the proof of the Theorem 1, it is sufficient to find a function $V$ such that (39), (40) hold. Take $V(z)=z^{\top} W z$, where $W$ is the solution to (26), and suppose that $i=\operatorname{argmin}_{j \in \mathcal{M}} z^{\top} R_{j} z$. Then

$$
\begin{aligned}
\mathbb{E}_{z_{k}}\left[V\left(z_{k+1}\right)\right]-V\left(z_{k}\right) & =z_{k}^{\top}\left[J_{\mathrm{d}_{1}(i)}^{\top} E(W) J_{\mathrm{d}_{1}(i)}-W\right] z_{k}, \\
& =-z_{k}^{\top}\left[\sum_{j=1, j \neq i}^{m_{D}} c_{i j}\left(R_{j}-R_{i}\right)+Q_{i}\right] z_{k}
\end{aligned}
$$

where $Q_{i}>0$. Since $i=\operatorname{argmin}_{j \in \mathcal{M}} z_{k}^{\top} R_{j} z_{k} \quad$ we have $z_{k}^{\top}\left[\sum_{j=1, j \neq i}^{m D} c_{i j}\left(R_{j}-R_{i}\right)\right] z_{k} \geq 0$ for every $z_{k} \in \mathfrak{R}^{n_{x}}$. Therefore from (45), we conclude that $V$ satisfies (40). It is also clear that $V$ satisfies (39), which concludes the proof.

Proof of Theorem 7. If, for a given $z_{k} \neq 0, i \in \mathcal{M}_{D}$ is such that $i=\operatorname{argmin}_{j \in \mathcal{M}_{D}} z_{k}^{\top} R_{j} z_{k}$, which is equivalent to

$$
g_{j}^{i}\left(z_{k}\right) \geq 0, \quad \forall_{j \in \mathcal{M}_{D}-\{i\}},
$$

then we have that $z_{k+1}=e^{A k_{k}} J_{\mathrm{d}(i)} z_{k}$, in which case (28) boils down to

$$
g_{0}^{i}\left(z_{k}\right)>0 .
$$

The fact that the dynamic protocol is regular implies that there exists at least one $y$ such that (46) holds with strict inequality. Then, a straightforward adaptation of the lossless S-Procedure theorem provided in [13, Theorem 2] to handle strict inequalities in the objective function $g_{0}^{i}(y)$ assures that, under Condition (29), (47) holds if and only if there exist $c_{i j} \geq 0, j \in \mathcal{M}_{D}-\{i\}$ such that:

$$
g_{0}^{i}(y)-\sum_{j \in \mathcal{M}_{D}-\{i\}} c_{i j} g_{j}^{i}(y)>0, \quad \forall \forall_{y \in \mathbb{R}^{n_{x}}-\{0\}}
$$

The result then follows by noticing that (48) is equivalent to (26). The fact that (29) holds if $m_{D}=2$ (in which case there is only one quadratic constraint) follows from [13, Theorem 3].

Proof of Theorem 8. We first prove that, if there exists $P>0$ such that

$$
\begin{aligned}
& \int_{0}^{\gamma}\left(e^{A P h}+H(h) L_{k} \Psi_{i} C_{P}\right)^{\top} P \ldots\left(e^{A P h}+H(h) L_{k} \Psi_{i} C_{P}\right) \mu(d h) \\
& \quad+\sum_{j=1, j \neq i}^{m} c_{i j}\left(C_{P}^{\top} S_{j} C_{P}-C_{P}^{\top} S_{i} C_{P}\right)-P<0
\end{aligned}
$$

then (26) holds for (12) with Matrices (33). Note that we can assume that $\mathrm{d}_{1}(i)=i, \forall_{i \in \mathcal{M}}$ since, if this is not the case, we can relabel the sensor nodes in such a way that this holds. For $A, J_{i}$ given by (33) we have:

$$
e^{A h} J_{i}=\left[\begin{array}{cc}
e^{A P h}+H(h) L_{k} \Psi_{i} C_{P} & 0 \\
L_{k} \Psi_{i} C & 0
\end{array}\right] .
$$

Using this expression and considering $W=\operatorname{diag}\left(\left[P \varepsilon I_{n_{P}}\right]\right)$ in (26), where $P$ satisfies (49) and $\varepsilon$ is a given positive constant, we have:

$$
\begin{aligned}
& \int_{0}^{\gamma}\left[\begin{array}{cc}
B(h) & 0 \\
0 & 0
\end{array}\right] \mu(d h)-\left[\begin{array}{cc}
P & 0 \\
0 & \varepsilon I_{n_{P}}
\end{array}\right] \\
& \quad+\sum_{j=1, j \neq i}^{m} c_{i j}\left[\begin{array}{cc}
C_{P}^{\top} S_{j} C_{P}-C_{P}^{\top} S_{i} C_{P} & 0 \\
0 & 0_{n_{P}}
\end{array}\right]<0, \quad \forall_{i \in \mathcal{M}}
\end{aligned}
$$

where

$$
\begin{aligned}
B(h)= & \varepsilon\left(L_{k} \Psi_{i} C_{P}\right)^{\top}\left(L_{k} \Psi_{i} C_{P}\right) \\
& +\left(e^{A P h}+H(h) L_{k} \Psi_{i} C_{P}\right)^{\top} P\left(e^{A P h}+H(h) L_{k} \Psi_{i} C_{P}\right) .
\end{aligned}
$$

From this expression we conclude that, if (49) holds, then (50) holds for sufficiently small $\varepsilon$. Set $L_{k}=H\left(h_{k}\right)^{-1} P^{-1} M$ for a $n_{P} \times m$ matrix. Then, (49) can be written as:

$$
\begin{gathered}
F(P)+D M \Psi_{i} C_{P}+\left(D M \Psi_{i} C_{P}\right)^{\top}+C_{P}^{\top} M P^{-1} M C_{P} \\
+\sum_{j=1, j \neq i}^{m} c_{i j}\left(C_{P}^{\top} S_{j} C_{P}-C_{P}^{\top} S_{i} C_{P}\right)-P<0, \forall_{i \in \mathcal{M}} .
\end{gathered}
$$

If we let $Y>0$ be such that $M^{\top} P^{-1} M<Y$, which, applying the Shur complement, can be seen to be equivalent to (36), we see that, if (35) holds, then (51) holds, which concludes the proof.

Proof of Theorem 10. The proof is obtained by following the same steps of Theorem 1 and by noticing that, using a similar reasoning to (44), one obtains:

$$
\mathbb{E}_{z_{k}}\left[V\left(z_{k+1}\right)\right] \leq \sum_{l=1}^{n_{q}} w_{l}\left(K_{\mathrm{d}_{1}(i)}^{l} \top^{\top}\left(\sum_{j=1}^{m_{D}} p_{j i} E\left(R_{j}\right)\right) K_{\mathrm{d}_{1}(i)}^{l}\right) .
$$


Proof of Theorem 11. The proof is obtained by following the same steps of Theorem 6 and by noticing that, in (45)

$$
\mathbb{E}_{z_{k}}\left[V\left(z_{k+1}\right)\right]=\sum_{l=1}^{n_{q}} w_{l}\left(K_{\mathrm{d}_{1}(i)}^{l}\right)^{\top} E(W) K_{\mathrm{d}_{1}(i)}^{l}
$$

\section{REFERENCES}

1. Walsh, G. and H. Ye, "Scheduling of networked control systems," IEEE Control Syst. Mag., Vol. 21, No. 1, pp. 57-65 (2001).

2. Nesic, D. and A. Teel, "Input-output stability properties of networked control systems," IEEE Trans. Autom. Control, Vol. 49, No. 10, pp. 1650-1667 (2004).

3. Tabbara, M., D. Nesic, and A. Teel, "Stability of wireless and wireline networked control systems," IEEE Trans. Autom. Control, Vol. 52, No. 9, pp. 1615-1630 (2007).

4. Donkers, M., L. Hetel, W. Heemels, N. van de Wouw, and M. Steinbuch, "Stability analysis of networked control systems using a switched linear systems approach," In R. Majumdar and P. Tabuada (Eds) Hybrid systems: Computation and control, ser. Lecture Notes in Computer Science, Springer-Verlag, Berlin Heidelberg, pp. 150164 (2009).

5. Hespanha, J. P. and A. R. Teel, "Stochastic impulsive systems driven by renewal processes," In 17th international symposium on mathematical theory of networks and systems (MTN06), Kyoto, Japan, pp. 608-618 (2006).

6. Antunes, D., J. P. Hespanha, and C. Silvestre, "Stability of impulsive systems driven by renewal processes," In American Control Conference, 2009. ACC '09., St Louis, MO, pp. 4032-4037 (2009).

7. Donkers, M., W. Heemels, D. Bernardini, A. Bemporad, and V. Shneer, "Stability analysis of stochastic networked control systems," In American Control Conference (ACC), 2010, Baltimore, MA, pp. 3684-3689 (2010).

8. Dacic, D. and D. Nesic, "Observer design for wired linear networked control systems using matrix inequalities," Automatica, Vol. 44, No. 11, pp. 2840-2848 (2008).

9. Antunes, D., J. P. Hespanha, and C. Silvestre, "Stochastic networked control systems with dynamic protocols," In American Control Conference (ACC), 2011, 29 2011July 1, San Francisco, CA, pp. 1686-1691 (2011).

10. Tabbara, M. and D. Nesic, "Input output stability of networked control systems with stochastic protocols and channels," IEEE Trans. Autom. Control, Vol. 53, No. 5, pp. 1160-1175 (2008).

11. Antunes, D., J. P. Hespanha, and C. Silvestre, "Control of impulsive renewal systems: Application to direct design in networked control," In Proc. of the 48th Conf. on Decision and Contr., pp. 6882-6887 (2009).
12. Costa, O. L. V., M. Fragoso, and R.P. Marques, DiscreteTime Markov Jump Linear Systems. Springer-Verlag, London (2005).

13. Jonsson, U. T., "A lecture on the s-procedure," Royal Institute of Technology, Tech. Rep., 2001, available at http://www.math.kth.se/uj/5B5746/Lecture.ps.

14. You, K. and L. Xie, "Minimum data rate for mean square stabilization of discrete lti systems over lossy channels," IEEE Trans. Autom. Control, Vol. 55, No. 10, pp. 23732378 (2010).

15. Liu, T., H. Zhang, and Q. Chen, "Robust h2 filtering for discrete-time Markovian jump linear systems," Asian J. Control, Vol. 14, No. 6, pp. 1599-1607 (2012).

16. Ebihara, Y., "Periodically time-varying memory statefeedback for robust h2 control of uncertain discrete-time linear systems," Asian J. Control, Vol. 15, No. 2, pp. 409-419 (2013).

17. Xie, W., "Multi-objective H2/L2 performance controller synthesis for LPV systems," Asian J. Control, Vol. 14, No. 5, pp. 1273-1281 (2012).

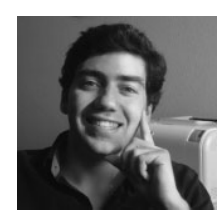

Duarte Antunes was born in Viseu, Portugal, in 1982. He received the Licenciatura in Electrical and Computer Engineering from the Instituto Superior Técnico (IST), Lisbon, in 2005. He did his PhD from 2006 to 2011 in the research field of Automatic Control at the Institute for Systems and Robotics, IST, Lisbon. From 2011 to 2013 he held a postdoctoral position at the Eindhoven University of Technology (TU/e). He is currently University Lecturer at the Department of Mechanical Engineering of TU/e. His research interests include Networked Control Systems, Stochastic Control, Renewal Systems, Multi-rate and Sampled-Data Systems, Dynamic Programming, and Systems Biology.

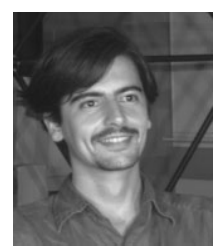

João P. Hespanha was born in Coimbra, Portugal, in 1968. He received the Licenciatura in electrical and computer engineering from the Instituto Superior Técnico, Lisbon, Portugal in 1991 and the $\mathrm{Ph} . \mathrm{D}$. degree in electrical engineering and applied science from Yale University, New Haven, Connecticut in 1998. From 1999 to 2001, he was Assistant Professor at the University of Southern California, Los Angeles. He moved to the University of California, Santa Barbara in 2002, where he currently holds Professor position with the Department of Electrical and Computer Engineering. Prof. Hespanha is the Chair of the Department of Electrical and Computer Engineering; Director for the Center for Control, Dynamical-systems, and Computation (CCDC); and a member of the Executive Committee for the Institute for Collaborative Biotechnologies (ICB). Dr. Hespanha is the 
recipient of the Yale University's Henry Prentiss Becton Graduate Prize for exceptional achievement in research in Engineering and Applied Science, a National Science Foundation CAREER Award, the 2005 best paper award at the 2nd Int. Conf. on Intelligent Sensing and Information Processing, the 2005 Automatica Theory/Methodology best paper prize, the 2006 George S. Axelby Outstanding Paper Award, and the 2009 Ruberti Young Researcher Prize. Dr. Hespanha is a Fellow of the IEEE and an IEEE distinguished lecturer since 2007. His current research interests include hybrid and switched systems; multi-agent control systems; distributed control over communication networks (also known as networked control systems); the use of vision in feedback control; stochastic modeling in biology; and network security.

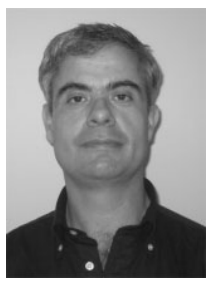

Carlos Silvestre received the Licenciatura degree in Electrical Engineering from the Instituto Superior Tecnico (IST) of Lisbon, Portugal, in 1987 and the M.Sc. degree in Electrical Engineering and the Ph.D. degree in Control Science from the same school in 1991 and 2000, respectively. In
2011 he received the Habilitation in Electrical Engineering and Computers also from IST. Since 2000, he is with the Department of Electrical Engineering of the Instituto Superior Tecnico, where he is currently Associate Professor of Control and Robotics in leave. Since 2012 he is Associate Professor of the Department o Electrical and Computers Engineering of the Faculty of Science and Technology of the University of Macau. Over the past years, he has conducted research on the subjects of navigation guidance and control of air and underwater robots. His research interests include linear and nonlinear control theory, coordinated control of multiple vehicles, gain scheduled control, integrated design of guidance and control systems, inertial navigation systems, and mission control and real time architectures for complex autonomous systems with applications to unmanned air and underwater vehicles. 